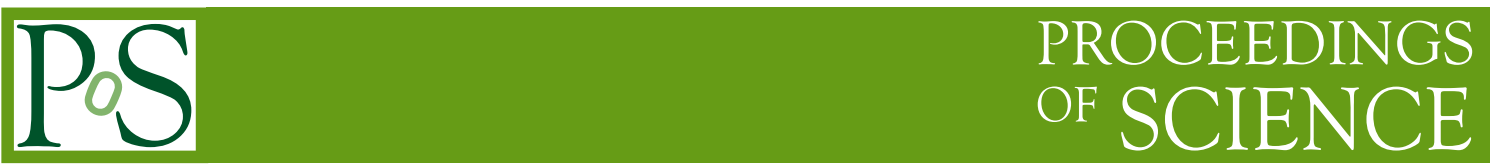

\title{
Rare B-Decays: An Update from the ATLAS Experiment
}

\author{
Ann-Kathrin Perrevoort, on behalf of the ATLAS Collaboration ${ }^{a, *}$ \\ ${ }^{a}$ Nikhef, \\ Science Park 105, 1098 XG Amsterdam, The Netherlands \\ E-mail: ann-kathrin.perrevoort@cern.ch
}

Rare flavour-changing neutral current processes are sensitive probes for physics beyond the Standard Model. The ATLAS experiment at the Large Hadron Collider (LHC) has performed a measurement of the branching fraction of $B_{s}^{0} \rightarrow \mu^{+} \mu^{-}$decays and a search for $B^{0} \rightarrow \mu^{+} \mu^{-}$ decays with data collected in 2015 and 2016, in combination with a previous result by ATLAS on data collected in 2011 and 2012. The results are combined with recent results by the CMS and LHCb experiments. The angular decay distributions of $B^{0} \rightarrow K^{* 0} \mu^{+} \mu^{-}$decays are measured with $20.3 \mathrm{fb}^{-1}$ of data collected in 2012. For both analyses, the expected improvement in sensitivity and precision is estimated for the High-Luminosity LHC.

BEAUTY2020

21-24 September 2020

Kashiwa, Japan (online)

${ }^{*}$ Speaker 


\section{Introduction}

The flavour-changing neutral current (FCNC) processes, $b \rightarrow s \mu^{+} \mu^{-}$, are mediated in the Standard Model (SM) via loop or box diagrams and thus occur at low rates. This turns rare $B$ meson decays into sensitive probes of physics beyond the SM, as such processes could enhance the branching fractions and modify angular decay distributions.

The ATLAS experiment [1] at the Large Hadron Collider (LHC) measures the branching fractions of the rare decays $B^{0} \rightarrow \mu^{+} \mu^{-}$and $B_{s}^{0} \rightarrow \mu^{+} \mu^{-}$as well as angular decay distributions of $B^{0} \rightarrow K^{* 0} \mu^{+} \mu^{-}$. The former measurement is combined with recent results from the CMS and LHCb experiments. For both measurements, the expected sensitivities for the High-Luminosity LHC upgrade (HL-LHC) [2] are estimated.

\section{Measurement of the branching fractions of $B_{(s)}^{0} \rightarrow \mu^{+} \mu^{-}$}

The ATLAS experiment has performed a measurement of the branching fractions $\mathcal{B}\left(B^{0} \rightarrow\right.$ $\left.\mu^{+} \mu^{-}\right)$and $\mathcal{B}\left(B_{s}^{0} \rightarrow \mu^{+} \mu^{-}\right)$using $36.2 \mathrm{fb}^{-1}$ of $p p$ collision data at a centre-of-mass energy of $\sqrt{s}=13 \mathrm{TeV}$ collected in 2015 and 2016 (early LHC Run 2) [3], and has combined the results with the Run 1 measurement based on $25 \mathrm{fb}^{-1}$ data at a $\sqrt{s}$ of 7 and $8 \mathrm{TeV}$ [4].

The Run 2 data is collected using a two-level trigger system. In the hardware-based first-level trigger system, dimuon events passing transverse momentum thresholds $\left(p_{\mathrm{T}}^{\mu_{1}}, p_{\mathrm{T}}^{\mu_{2}}\right)$ of $(4 \mathrm{GeV}, 6 \mathrm{GeV})$ are selected. The software-based high-level trigger requires in addition a dimuon invariant mass $m_{\mu \mu}$ between $4 \mathrm{GeV}$ and $8.5 \mathrm{GeV}$. Further selections are based on the quality of the reconstructed muons and the reconstructed $B_{(s)}$ vertex. Events with $4.766 \mathrm{GeV}<m_{\mu \mu}<5.966 \mathrm{GeV}$ are considered in the analysis, of which the range $5.166 \mathrm{GeV}<m_{\mu \mu}<5.526 \mathrm{GeV}$ is defined as signal region.

The contribution of continuum combinatorial background originating from muons of uncorrelated hadron decays is reduced by the means of a boosted decision tree (BDT). The BDT takes 15 variables into account, and is trained and tested on data sidebands and simulated signal events. The $m_{\mu \mu}$ distributions of partially reconstructed $b \rightarrow \mu^{+} \mu^{-} X$ decays have a tail reaching into the signal region, and therefore, are taken into account in the fit. A further background component stems from $B_{(s)} \rightarrow h h^{\prime}$ decays with the hadrons $h^{\left({ }^{\prime}\right)}=\pi^{ \pm}, K^{ \pm}$being mis-identified as muon candidates. This background peaks in the signal region and is reduced by a tight muon quality selection.

The $B_{(s)}^{0} \rightarrow \mu^{+} \mu^{-}$branching fractions are measured relative to the abundant and well-measured reference channel $B^{ \pm} \rightarrow J / \psi\left(\rightarrow \mu^{+} \mu^{-}\right) K^{ \pm}$. The yield in the reference channel is determined in an unbinned maximum likelihood fit to the invariant mass $m_{J / \psi K^{ \pm}}$of the $\mu^{+} \mu^{-} K^{ \pm}$system, while the efficiency relative to the signal decay $B_{(s)}^{0} \rightarrow \mu^{+} \mu^{-}$is taken from MC simulations.

The signal yield is extracted in an simultaneous unbinned maximum likelihood fit to the $m_{\mu \mu}$ distribution in four BDT intervals of constant signal efficiency. The fitted signal and background contributions in the highest BDT interval are shown in Fig. 1 on the left. Due to the limited mass resolution, the peaks of $B^{0} \rightarrow \mu^{+} \mu^{-}$and $B_{s}^{0} \rightarrow \mu^{+} \mu^{-}$overlap and are statistically separated in the fit. Using the Run 2 data set, $80 \pm 22 B_{s}^{0} \rightarrow \mu^{+} \mu^{-}$and $-12 \pm 20 B^{0} \rightarrow \mu^{+} \mu^{-}$events are extracted. The result is in agreement with the SM predictions. The branching fraction of the $B_{s}^{0} \rightarrow \mu^{+} \mu^{-}$ decay obtained in a Neyman construction is $\mathcal{B}\left(B_{s}^{0} \rightarrow \mu^{+} \mu^{-}\right)=\left(3.21_{-0.91}^{+0.96} \text { (stat. }\right)_{-0.30}^{+0.49}($ syst. $\left.)\right) \times 10^{-9}$. For $B^{0} \rightarrow \mu^{+} \mu^{-}$, an upper limit is set to $\mathcal{B}\left(B^{0} \rightarrow \mu^{+} \mu^{-}\right)<4.3 \times 10^{-10}$ at $95 \%$ confidence level 

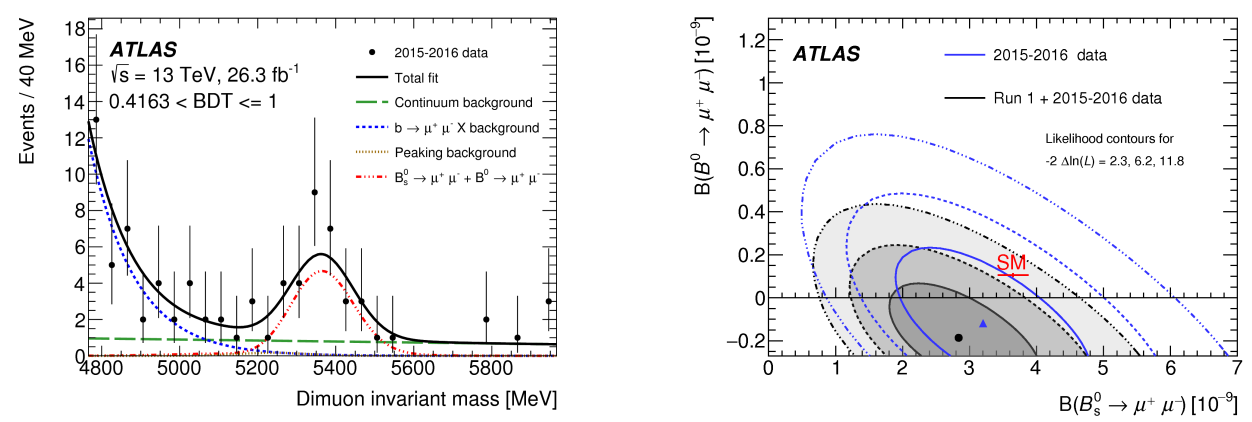

Figure 1: Results from the measurement of $\mathcal{B}\left(B_{(s)}^{0} \rightarrow \mu^{+} \mu^{-}\right)$[3]. Left: Distributions of $m_{\mu \mu}$ in the unblinded data in the highest BDT interval. Superimposed is the result of the maximum likelihood fit; Right: Likelihood contours for the simultaneous fit to $\mathcal{B}\left(B_{s}^{0} \rightarrow \mu^{+} \mu^{-}\right)$and $\mathcal{B}\left(B^{0} \rightarrow \mu^{+} \mu^{-}\right)$, for values of $-2 \Delta \ln (\mathcal{L})$ equal to $2.3,6.2,11.8$.
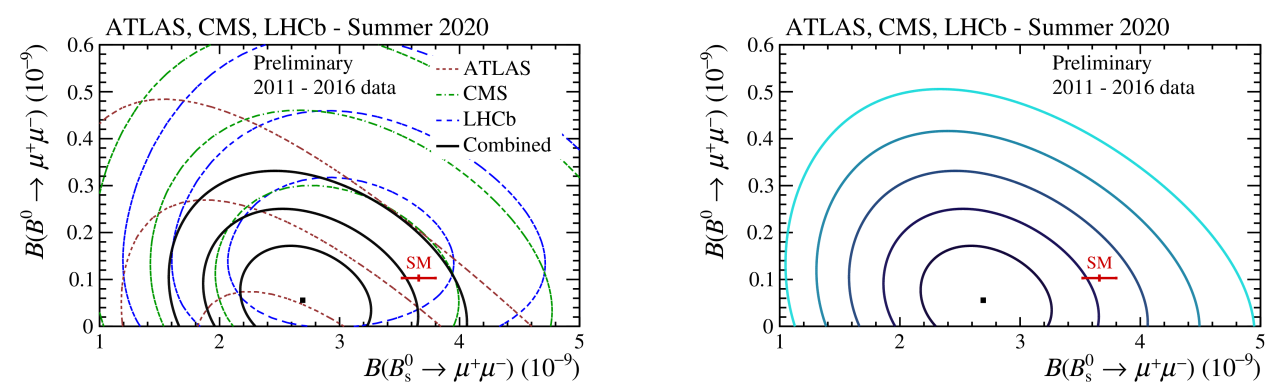

Figure 2: Likelihood contours for the results of $\mathcal{B}\left(B_{(s)}^{0} \rightarrow \mu^{+} \mu^{-}\right)$for the ATLAS, CMS, and LHCb experiments as well as their combination [6]. Left: Contours correspond to values of $-2 \Delta \ln (\mathcal{L})=2.3,6.2$, and 11.8; Right: Contours of the combination for values of $-2 \Delta \ln (\mathcal{L})=2.3,6.2,11.8,19.3$, and 30.2.

(CL). The likelihood functions from the Run 2 result are combined with the Run 1 results, yielding $\mathcal{B}\left(B_{s}^{0} \rightarrow \mu^{+} \mu^{-}\right)=\left(2.8_{-0.7}^{+0.8}\right) \times 10^{-9}$, and an upper limit for $B^{0} \rightarrow \mu^{+} \mu^{-}$of $\mathcal{B}\left(B^{0} \rightarrow \mu^{+} \mu^{-}\right)<$ $2.1 \times 10^{-10}$ at $95 \% \mathrm{CL}$. The likelihood contours are shown in Fig. 1 on the right. The results differ by 2.4 standard deviations $\sigma$ from the SM [5] in the $\mathcal{B}\left(B^{0} \rightarrow \mu^{+} \mu^{-}\right)-\mathcal{B}\left(B_{s}^{0} \rightarrow \mu^{+} \mu^{-}\right)$plane.

\subsection{Combination of ATLAS, CMS and LHCb results}

The results on $\mathcal{B}\left(B_{s}^{0} \rightarrow \mu^{+} \mu^{-}\right)$of the ATLAS experiment are consistent with results obtained by the CMS and LHCb experiments with data collected between 2011 and 2016 [6] (see Fig. 2). The results of the three experiments are combined. A $B_{s}^{0} \rightarrow \mu^{+} \mu^{-}$branching fraction of $\mathcal{B}\left(B_{s}^{0} \rightarrow\right.$ $\left.\mu^{+} \mu^{-}\right)=\left(2.69_{-0.35}^{+0.37}\right) \times 10^{-9}$ is measured and an upper limit on the $B^{0} \rightarrow \mu^{+} \mu^{-}$branching fraction is set at $\mathcal{B}\left(B_{s}^{0} \rightarrow \mu^{+} \mu^{-}\right)<1.9 \times 10^{-10}$ at $95 \% \mathrm{CL}$. This corresponds to a deviation from the SM predictions by $2.1 \sigma$.

\section{Angular analysis of $B^{0} \rightarrow K^{* 0} \mu^{+} \mu^{-}$}

An angular analysis of the decay $B^{0} \rightarrow K^{* 0}\left(\rightarrow K^{+} \pi^{-}\right) \mu^{+} \mu^{-}$is performed with the ATLAS experiment using $20.3 \mathrm{fb}^{-1}$ of $p p$ collision data at $\sqrt{s}=8 \mathrm{TeV}$ collected in 2012 [7]. The angular 

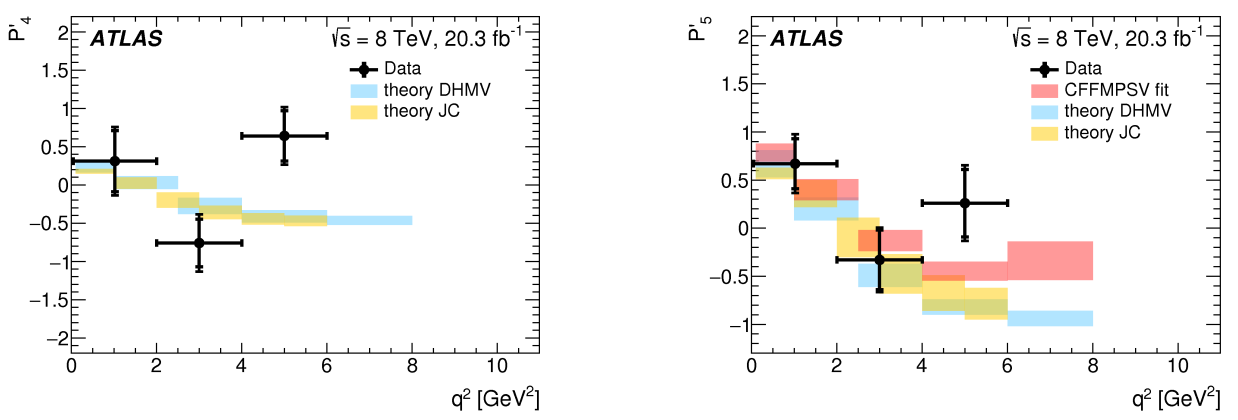

Figure 3: The measured values of $P_{4}^{\prime}$ and $P_{5}^{\prime}$ compared with predictions from the theoretical calculations [911]. Statistical and total uncertainties are shown for the data, i.e. the inner mark indicates the statistical uncertainty and the total error bar the total uncertainty. Figures taken from [7].

differential decay rate is described by three angles and the invariant dimuon mass squared, $q^{2}$. This analysis employs the same parametrisation as used by the LHCb experiment in terms of the optimised parameters $P_{i}^{\left({ }^{\prime}\right)}$ which minimise uncertainites from hadronic form factors [8]. A trigonometric folding of the angular variables is performed due to limited statistics.

In the event selection, trigger chains with one, two, or at least three muons are combined to ensure a high signal yield and a sensitivity down to low $q^{2}$. Events with two charged tracks compatible with an invariant $K \pi$ mass, $m_{K \pi}$, between $846 \mathrm{MeV}$ and $946 \mathrm{MeV}$ and two muons with $q^{2} \in[0.04,6.0] \mathrm{GeV}^{2}$, that have an invariant mass of the $K \pi \mu \mu$ system, $m_{K \pi \mu \mu}$, in the range of 5110 to $5700 \mathrm{MeV}$ are selected for analysis. Events with $q^{2}>6.0 \mathrm{GeV}^{2}$ are excluded to suppress the radiative tail from $B^{0} \rightarrow K^{* 0} J / \psi$ decays. A veto on the $D^{0}$ and $D_{(s)}^{+}$mass ranges is applied to diminish background contributions from $B^{0} \rightarrow D^{0} / D_{(s)}^{+} X$ decays. Further selections on the reconstructed vertex are applied to suppress combinatorial background.

An extended unbinned maximum-likelihood fit to $m_{K \pi \mu \mu}$ and the three angles is performed in six partially overlapping bins of $q^{2}$ yielding a total of $342 \pm 39$ signal events. All measured values are compatible with the SM predictions [9-11] within three standard deviations. A comparison of the measured values to theoretical predictions is exemplarily shown for $P_{4}^{\prime}$ and $P_{5}^{\prime}$ in Fig. 3. For these parameters, the overall largest deviations of $2.7 \sigma$ compared to the DHMV model [10] are observed in the $q^{2} \in[4.0,6.0] \mathrm{GeV}^{2}$ bin, consistent with results by the LHCb collaboration [8].

\section{Prospects for the High-Luminosity LHC}

For both analyses, the expected sensitivities for the HL-LHC upgrade are estimated in pseudoMC experiments based on the results from the Run 1 analyses $[12,13]$. In the case of $B_{(s)}^{0} \rightarrow \mu^{+} \mu^{-}$, also an estimate for the full Run 2 (2015-18) is given. It is assumed that the Run 1 analysis strategy is kept, and thus the offline selection and reconstruction efficiencies. The yield is adapted for the increase in the $B_{(s)}$ production cross-section due to the higher $\sqrt{s}$ (13 TeV in Run 2 and $14 \mathrm{TeV}$ in HL-LHC instead of $8 \mathrm{TeV}$ in Run 1) as well as the increase of the expected integrated luminosity ( $130 \mathrm{fb}^{-1}$ in Run 2, $3 \mathrm{ab}^{-1}$ in HL-LHC). In the Run 2 estimate, the respective trigger conditions and efficiencies are considered. In the HL-LHC case, three dimuon trigger scenarios are defined: a conservative working point with trigger thresholds $\left(p_{\mathrm{T}}^{\mu_{1}}, p_{\mathrm{T}}^{\mu_{2}}\right)$ of $(10 \mathrm{GeV}, 10 \mathrm{GeV})$, as 

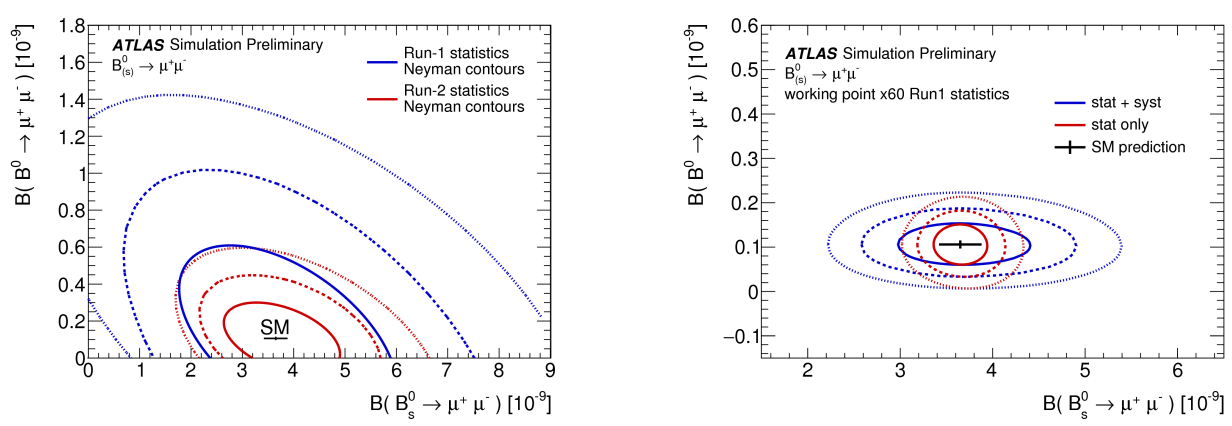

Figure 4: Comparison of the $68.3 \%, 95.5 \%$ and $99.7 \%$ confidence regions for the Run 1 and extrapolated Run 2 statistics (on the left) and for the intermediate working point of the HL-LHC extrapolation (on the right). Figures taken from [12].
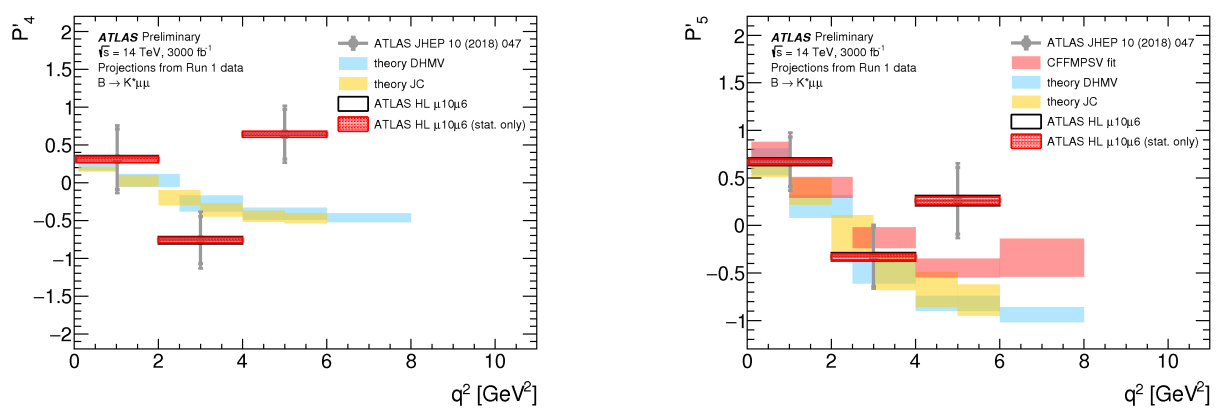

Figure 5: Projected ATLAS HL-LHC measurement precision in the $P_{4}^{\prime}$ and $P_{5}^{\prime}$ parameters for the intermediate working point compared to the ATLAS Run 1 results and theory predictions [9-11]. Figures taken from [13].

well as an intermediate $(6 \mathrm{GeV}, 10 \mathrm{GeV})$ and high-yield one $(6 \mathrm{GeV}, 6 \mathrm{GeV})$. Improvements on the mass resolution due to the HL-LHC ATLAS tracker upgrades are considered as well.

Both analyses will profit from the increased statistics and improved mass resolution. The expected likelihood contours for $\mathcal{B}\left(B_{s}^{0} \rightarrow \mu^{+} \mu^{-}\right)$centered around the SM expectation values in Run 2 and the HL-LHC (intermediate working point) are shown in Fig. 4. The improved mass resolution at the HL-LHC leads to a better separation of $B^{0} \rightarrow \mu^{+} \mu^{-}$and $B_{s}^{0} \rightarrow \mu^{+} \mu^{-}$decays. The projected measurement precision for the $P_{4}^{\prime}$ and $P_{5}^{\prime}$ in the HL-LHC (intermediate working point) are shown in Fig. 5.

\section{Summary}

FCNC processes $b \rightarrow s \mu^{+} \mu^{-}$are studied with the ATLAS experiment. A measurement of the branching fraction of the rare decay $B_{s}^{0} \rightarrow \mu^{+} \mu^{-}$and search for $B^{0} \rightarrow \mu^{+} \mu^{-}$on $25 \mathrm{fb}^{-1}$ plus $36.2 \mathrm{fb}^{-1}$ of $p p$ collision data collected between 2011 and 2016 is well in agreement with SM predictions. The result is combined with measurements by the CMS and LHCb experiments. The combined results deviate from the SM predictions by $2.1 \sigma$.

The results of the analysis of the angular decay distributions of $B^{0} \rightarrow K^{* 0} \mu^{+} \mu^{-}$on $20.3 \mathrm{fb}^{-1}$ of Run 1 data are found to be consistent with SM predictions with the largest deviation of $2.7 \sigma$ observed in the parameters $P_{4}^{\prime}$ and $P_{5}^{\prime}$ in the $q^{2} \in[4.0,6.0] \mathrm{GeV}^{2}$ bin. 
Both analyses will greatly profit from the increased luminosity and improved mass resolution in the HL-LHC.

\section{References}

[1] ATLAS Collaboration, The ATLAS Experiment at the CERN Large Hadron Collider, JINST 3 S08003 (2008).

[2] G. Apollinari, I. Béjar Alonso, O. Brüning, P. Fessia, M. Lamont, L. Rossi, and L. Tavian, High-Luminosity Large Hadron Collider (HL-LHC): Technical Design Report V. 0.1, CERN2017-007-M, https://cds.cern.ch/record/2284929.

[3] ATLAS Collaboration, Study of the Rare Decays of $B_{s}^{0}$ and $B^{0}$ Mesons into Muon Pairs Using Data Collected during 2015 and 2016 with the ATLAS Detector, JHEP 04098 (2019) [hep-ex/1812.03017].

[4] ATLAS Collaboration, Study of the Rare Decays of $B_{s}^{0}$ and $B^{0}$ into Muon Pairs from Data Collected during the LHC Run 1 with the ATLAS Detector, Eur.Phys.J.C 769513 (2016) [hep-ex/1604.04263].

[5] M. Beneke, C. Bobeth, and R. Szafron, Power-Enhanced Leading-Logarithmic QED Corrections to $B_{q} \rightarrow \mu^{+} \mu^{-}$, JHEP 10232 (2019) [hep-ph/1908.07011].

[6] ATLAS, CMS and LHCb Collaborations, Combination of the ATLAS, CMS and LHCb Results on the $B_{(s)}^{0} \rightarrow \mu^{+} \mu^{-}$Decays.", ATLAS-CONF-2020-049, CMS PAS BPH-20-003, LHCbCONF-2020-002, http://cds.cern.ch/record/2727207.

[7] ATLAS Collaboration, Angular Analysis of $B_{d}^{0} \rightarrow K^{*} \mu^{+} \mu^{-}$Decays in pp Collisions at $\sqrt{s}=8$ TeV with the ATLAS Detector, JHEP 10047 (2018) [hep-ex/1805.04000].

[8] LHCb Collaboration, Angular Analysis of the $B^{0} \rightarrow K^{* 0} \mu^{+} \mu^{-}$Decay using $3 \mathrm{fb}^{-1}$ of Integrated Luminosity, JHEP 02104 (2016) [hep-ex/1512.04442].

[9] M. Ciuchini, M. Fedele, E. Franco, et al., $B \rightarrow K^{*} \ell^{+} \ell^{-}$Decays at Large Recoil in the Standard Model: A Theoretical Reappraisal, JHEP 06116 (2016) [hep-ph/1512.07157].

[10] S. Descotes-Genon, L. Hofer, J. Matias, et al., On the Impact of Power Corrections in the Prediction of $B \rightarrow K^{*} \mu^{+} \mu^{-}$Observables, JHEP 12125 (2014) [hep-ph/1407.8526].

[11] S. Jäger, and J. Martin Camalich, Reassessing the Discovery Potential of the $B \rightarrow K^{*} \ell^{+} \ell^{-}$ Decays in the Large-Recoil Region: SM Challenges and BSM Opportunities, Phys. Rev. D 93 014028 (2016) [hep-ph/1412.3183].

[12] ATLAS Collaboration, Prospects for the $\mathcal{B}\left(B_{(s)}^{0} \rightarrow \mu^{+} \mu^{-}\right)$Measurements with the ATLAS Detector in the Run 2 and HL-LHC Data Campaigns, ATL-PHYS-PUB-2018-005, http://cdsweb.cern.ch/record/2317211.

[13] ATLAS Collaboration, $B_{d}^{0} \rightarrow K^{* 0} \mu \mu$ Angular Analysis Prospects with the Upgraded ATLAS Detector at the HL-LHC, ATL-PHYS-PUB-2019-003, http://cdsweb.cern.ch/record/2654519. 